\title{
GLOBAL CLASSICAL SOLUTIONS OF THE VLASOV-DARWIN SYSTEM FOR SMALL INITIAL DATA*
}

\author{
MARTIN SEEHAFER ${ }^{\dagger}$
}

\begin{abstract}
A global-in-time existence theorem for classical solutions of the Vlasov-Darwin system is given under the assumption of smallness of the initial data. Furthermore it is shown that in case of spherical symmetry the system degenerates to the relativistic Vlasov-Poisson system.
\end{abstract}

Key words. Relativistic Vlasov-Darwin system; global classical solutions; collisionless plasma

AMS subject classifications. 35F20, 82D10

\section{Introduction}

Kinetic models play an increasingly important role in todays plasma physics. On the one hand, much effort has been taken to deepen our analytical understanding of some problems where no other description seems to be adequate. On the other hand progress has also been achieved, especially with numerical simulations (see, e.g., [12]).

In the kinetic picture, the particle distribution of a one-species plasma is described by a time dependent density function $f(t, x, p)$ on phase space. If collisions of the particles are neglected and a relativistic model is used, then $f$ is subject to the transport equation

$$
\partial_{t} f+v(p) \cdot \nabla_{x} f+K(t, x) \cdot \nabla_{p} f=0
$$

with force term $K=E+v \times B$. Here $E$ and $B$ denote the electric and the magnetic field respectively and the relativistic velocity is given by

$$
v(p)=\frac{1}{\sqrt{1+|p|^{2}}} p
$$

Note that all physical constants such as the speed of light or the rest mass of the particles have been set equal to unity.

Equation (1.1) is usually called the Vlasov equation. Expressions for the charge and current densities $\rho$ and $j$ in terms of the phase space density $f$ are given by

$$
\rho(t, x)=\int f(t, x, p) d p, \quad j(t, x)=\int f(t, x, p) v(p) d p .
$$

To obtain a self-consistent closed system one has to take into account how the ensemble modeled by the density $f$ creates the fields $E$ and $B$. Usually this is done with the full system of Maxwell's equations, but numerical difficulties of simulations of that system have stimulated a search for alternatives (see [4]). The present paper deals with what is known as the Darwin approximation. Here the electric field is split into a transverse and a longitudinal component as follows:

$$
E=E_{L}+E_{T}, \quad \nabla \times E_{L}=0, \quad \nabla \cdot E_{T}=0 .
$$

*Received: February 25, 2008; accepted (in revised version): August 1, 2008. Communicated by Norbert Mauser.

${ }^{\dagger}$ University of Bayreuth, Department of Mathematics, D-95440 Bayreuth, Germany (martin. seehafer@uni-bayreuth.de). 
In the evolution part of the Maxwell equations the transverse part of the electric field is neglected, resulting in

$$
\begin{array}{rr}
\partial_{t} E_{L}-\nabla \times B=-j, & \nabla \cdot E_{L}=\rho \\
\partial_{t} B+\nabla \times E_{T}=0, & \nabla \cdot B=0 .
\end{array}
$$

The system consisting of the Equations (1.1) - ,(1.2),(1.3),(1.4),(1.5), and (1.6) is called the Vlasov-Darwin system. The main feature of this system is that the field equations are elliptic which in particular facilitates a numerical treatment since a time integration step, which is needed to solve the Maxwell system can be avoided here (cf. [12]). The justification of the model seems possible in the case where the particle velocities are not too fast when compared to the speed of light.

Up to now there are few mathematical results known for this system. In 2003, Benachour et al. [3] proved an existence theorem for small initial data: this assumption implies global-in-time existence of weak solutions of the Cauchy problem. Later Pallard [9] removed the smallness assumption and added a result about solvability of the Cauchy problem in a classical sense: for a given initial datum $f_{0} \in C_{c}^{2}\left(\mathbb{R}^{6}\right)$ there exists $T>0$ and a classical solution $f:\left[0, T\left[\times \mathbb{R}^{6} \rightarrow \mathbb{R}\right.\right.$ of the Vlasov-Darwin systems satisfying $f(0)=f_{0}$.

In the main part of the paper we present a result which is well known for the Vlasov-Poisson system (VP), the Relativistic Vlasov-Maxwell system (RVM), and other related systems such as the Relativistic Vlasov-Poisson system or the spherically symmetric Vlasov-Einstein system (cf. $[1,2,7,11]$ ) but seems to be new for the Vlasov-Darwin system. We consider classical solutions of the Cauchy problem and show that these exist for all times if the initial data are chosen to be sufficiently small. The precise statement of our result is contained in the next section, where we also formulate three propositions which are used to prove the theorem. Sections 3-6 are devoted to proofs. In section 7 we take a look at spherically symmetric solutions. First it is shown that any symmetry of the initial datum with respect to an orthogonal transformation is preserved for all times. This allows the conclusion that in the case of spherical symmetry, the VD system reduces to the well known relativistic VlasovPoisson system. In this case the solutions are global in time as well [6].

\section{Results}

Before presenting the main result of the present paper (which is formulated as the following theorem) we fix some notation. Let $R_{0}, P_{0}>0$ be fixed throughout the paper. For $r>0$, let $B_{r}:=B_{r}(0)=\left\{x \in \mathbb{R}^{3}:|x|<r\right\}$. Furthermore we must specify the set where the initial data are taken from. Let $C_{c}^{2}\left(\mathbb{R}^{n}\right)$ denote the space of twice continuously differentiable functions on $\mathbb{R}^{n}$ with compact support and

$$
\mathcal{D}:=\left\{f \in C_{c}^{2}\left(\mathbb{R}^{6}\right): f \geq 0,\|f\|_{\infty} \leq 1,\|\nabla f\|_{\infty} \leq 1, \operatorname{supp} f \subset B_{R_{0}}(0) \times B_{P_{0}}(0)\right\} .
$$

The Lebesgue space of square integrable functions is denoted by $L^{2}\left(\mathbb{R}^{3}\right)$ and $\mathbb{P}: L^{2}\left(\mathbb{R}^{3}\right) \rightarrow L^{2}\left(\mathbb{R}^{3}\right)$ is the projection on the divergence free part, which is discussed in the Appendix.

If $I$ is an interval and $g: I \times \mathbb{R}^{n} \rightarrow \mathbb{R}^{m}$, we denote the quantity $\sup _{x \in \mathbb{R}^{n}}|g(t, x)|$ by $\|g(t)\|_{\infty}$, and if $K \subset \mathbb{R}^{n}$, the expression $\|g(t)\|_{\infty, K}$ means $\sup _{x \in K}|g(t, x)|$.

THEOREM 2.1. There exists $\delta>0$ such that the classical solution of the VD system with initial datum $f_{0}$ in $\mathcal{D}$ satisfying $\left\|f_{0}\right\|_{\infty} \leq \delta$ exists globally in time. 
For the proof of this result the reformulation of the field equations of the VD system in terms of potentials $\Phi$ and $A$ given in [9] is used. Let

$$
\begin{array}{r}
\Delta \Phi=\rho, \quad \lim _{|x| \rightarrow \infty} \Phi(t, x)=0, \\
\Delta A=-\mathbb{P}(j), \quad \lim _{|x| \rightarrow \infty} A(t, x)=0 .
\end{array}
$$

Then the components of the electromagnetic field are

$$
E_{L}=\nabla \Phi, \quad B=\nabla \times A, \quad E_{T}=-\partial_{t} A,
$$

cf. $[9$, Lem2.3].

The proof of the theorem is given in section 6 . Sections $3,4,5$ contain the proofs of preliminary results, which are formulated in Propositions 2.3, 2.4 and 2.5. A certain decay condition plays a prominent role in the following.

Definition 2.2. A classical solution $f:\left[0, T\left[\times \mathbb{R}^{6} \rightarrow \mathbb{R}\right.\right.$ of the VD system is said to satisfy a free streaming condition with parameter $\alpha$ on an interval $[0, a]$ if

$$
\begin{array}{r}
\left\|E_{T}(t)\right\|_{\infty}+\left\|E_{L}(t)\right\|_{\infty}+\|B(t)\|_{\infty} \leq \alpha(1+t)^{-3 / 2} \\
\left\|\nabla E_{T}(t)\right\|_{\infty}+\left\|\nabla E_{L}(t)\right\|_{\infty}+\|\nabla B(t)\|_{\infty} \leq \alpha(1+t)^{-5 / 2}
\end{array}
$$

for all $t \in[0, a]$.

As for the RVM, there is a continuation criterion for solutions of the VD system, which says that solutions may be continued as long as the momentum support $\left\{p \in \mathbb{R}^{3}\right.$ : $\exists x, t: f(t, x, p) \neq 0\}$ remains bounded [9]. Using this criterion one can easily show that a solution which satisfies a condition such as the one above on its maximal interval of existence is indeed a global one.

We now discuss the main idea of the proof. The task will be to show that the free streaming condition implies decay of the source terms $\rho$ and $j$ as well as decay of the fields $E_{L}, E_{T}, B$. This will be done in the following two propositions.

Proposition 2.3. There exist $\alpha, C_{1}\left(R_{0}, P_{0}\right)>0$ such that for every solution of the $V D$ system with $f(0) \in \mathcal{D}$ which satisfies a free streaming condition on an interval $[0, a]$ with parameter $\alpha$, the following holds

$$
\|\rho(t)\|_{\infty}+\|j(t)\|_{\infty} \leq C_{1}|t|^{-3}, \quad\left\|\partial_{x} \rho(t)\right\|_{\infty}+\left\|\partial_{x} j(t)\right\|_{\infty} \leq C_{1} .
$$

Proposition 2.4. There exist $\alpha, C_{2}\left(R_{0}, P_{0}\right)>0$ such that for every solution of the $V D$ system with $f(0) \in \mathcal{D}$ which satisfies a free streaming condition on an interval $[0, a]$ with parameter $\alpha$, the following holds for $t \in[1, a]$

$$
\begin{array}{r}
\left\|E_{T}(t)\right\|_{\infty}+\left\|E_{L}(t)\right\|_{\infty}+\|B(t)\|_{\infty} \leq C_{2} t^{-9 / 5} \\
\left\|\nabla E_{T}(t)\right\|_{\infty}+\left\|\nabla E_{L}(t)\right\|_{\infty}+\|\nabla B(t)\|_{\infty} \leq C_{2} t^{-8 / 3}
\end{array}
$$

These estimates provide the main ingredient needed for the bootstrap argument in the proof of the theorem. It follows that a solution satisfying a free streaming condition decays asymptotically even faster. This is an important point for the global existence argument. To start this bootstrapping we need a further tool, which is given in the next proposition. If the initial datum is chosen sufficiently small, then the fields 
remain small for some time. This may be interpreted as a statement about continuous dependence on initial data.

Proposition 2.5. Let $\epsilon, T>0$ be given. Then there exists $\delta>0$ such that any classical solution $f$ of the VD system with $f(0) \in \mathcal{D}$ and $\|f(0)\|_{\infty} \leq \delta$ exists at least up to time $T$ and is such that

$$
\left\|E_{L}(t)\right\|_{\infty}+\left\|E_{T}(t)\right\|_{\infty}+\|B(t)\|_{\infty}+\left\|\nabla E_{L}(t)\right\|_{\infty}+\left\|\nabla E_{T}(t)\right\|_{\infty}+\|\nabla B(t)\|_{\infty} \leq \epsilon
$$

\section{Decay of the source terms}

For proving Proposition 2.3, we need the following

LEMma 3.1. Let $t>0, \xi \in C^{2}([0, t]), \xi(t)=\dot{\xi}(t)=0$ and let

$$
|\ddot{\xi}(s)| \leq c_{1}(s)+c_{2}(s)|\xi(s)|+c_{3}(s)|\dot{\xi}(s)|
$$

for $s \in[0, t]$, where $c_{1}, c_{2}, c_{3} \geq 0$ are continuous and $c_{3}$ is monotonically decreasing. Then

$$
|\xi(s)| \leq\left(\int_{s}^{t} \sigma c_{1}(\sigma) d \sigma\right) e^{\int_{s}^{t}\left(\sigma c_{2}(\sigma)+c_{3}(\sigma)\right) d \sigma}
$$

Proof. Define $z(s):=\int_{s}^{t}|\dot{\xi}(\tau)| d \tau$, so that $|\xi(s)| \leq z(s), \dot{z}(s)=-|\dot{\xi}(s)|, z(t)=\dot{z}(t)=$ 0 . Obviously

$$
\begin{aligned}
z(s) & =\int_{s}^{t}\left|\int_{\tau}^{t} \ddot{\xi}(\sigma) d \sigma\right| d \tau \\
& \leq \int_{s}^{t} \int_{\tau}^{t}|\ddot{\xi}(\sigma)| d \sigma d \tau
\end{aligned}
$$

so that by our assumptions

$$
\begin{aligned}
z(s) & \leq \int_{s}^{t} \int_{\tau}^{t} c_{1}(\sigma) d \sigma d \tau+\int_{s}^{t} \int_{\tau}^{t} c_{2}(\sigma) z(\sigma) d \sigma d \tau-\int_{s}^{t} \int_{\tau}^{t} c_{3}(\sigma) \dot{z}(\sigma) d \sigma d \tau \\
& =: I_{1}+I_{2}+I_{3}
\end{aligned}
$$

Changing the order of integration it follows that

$$
I_{1}=\int_{s}^{t} \int_{s}^{\sigma} c_{1}(\sigma) d \tau d \sigma \leq \int_{s}^{t} \sigma c_{1}(\sigma) d \sigma
$$

as well as

$$
I_{2}=\int_{s}^{t} \int_{s}^{\sigma} c_{2}(\sigma) z(\sigma) d \tau d \sigma \leq \int_{s}^{t} \sigma c_{2}(\sigma) z(\sigma) d \sigma .
$$

The integral $I_{3}$ can be estimated in the following way:

$$
I_{3} \leq \int_{s}^{t} c_{3}(\tau)\left(-\int_{\tau}^{t} \dot{z}(\sigma) d \sigma\right) d \tau=\int_{s}^{t} c_{3}(\tau) z(\tau) d \tau
$$


where the monotonicity of $c_{3}$ was used in the first step and the relation $z(t)=0$ in the second.

Hence the function $z$ satisfies the integral inequality

$$
z(s) \leq \int_{s}^{t} \sigma c_{1}(\sigma) d \sigma+\int_{s}^{t}\left[\sigma c_{2}(\sigma)+c_{3}(\sigma)\right] z(\sigma) d \sigma
$$

so that by Gronwall's Lemma

$$
|\xi(s)| \leq z(s) \leq\left(\int_{s}^{t} \sigma c_{1}(\sigma) d \sigma\right) e^{\int_{s}^{t}\left[\sigma c_{2}(\sigma)+c_{3}(\sigma)\right] d \sigma} .
$$

Proof. [Proof of Proposition 2.3.] The proof presented here is an adaptation of the corresponding argument for the Vlasov-Poisson system (cf. [10]). To get decay of $\rho$, a change of variables is performed in the integral defining it. The transformation determinant appearing can be shown to decay fast enough.

Let $f$ be a classical solution of the VD system with $f(0)=f^{\circ} \in \mathcal{D}$ and denote by $(X(s, t, x, p), P(s, t, x, p))$ the corresponding solution of the characteristic system

$$
\begin{aligned}
\dot{X}(s, t, x, p) & =v(P(s, t, x, p)), \\
\dot{P}(s, t, x, p) & =E(X(s, t, x, p), s)+v(P(s, t, x, p)) \times B(X(s, t, x, p), s)
\end{aligned}
$$

with initial condition $X(t, t, x, p)=x, P(t, t, x, p)=p$.

Then we have

$$
f(t, x, p)=f^{\circ}(X(0, t, x, p), P(0, t, x, p)) .
$$

If $|p| \leq P_{0}$, then by the free streaming condition,

$$
|P(t, 0, x, p)| \leq P_{0}+\int_{0}^{t}\left(\|E(s)\|_{\infty}+\|B(s)\|_{\infty}\right) d s \leq P_{0}+\alpha \int_{0}^{t}(1+s)^{-3 / 2} d s \leq P_{0}+2 \alpha .
$$

Hence for small enough $\alpha$ we may conclude that $|p| \geq P_{0}+1$ implies that $f(t, x, p)=0$.

Define

$$
\xi(s):=\partial_{p} X(s, t, x, p)-(s-t) D v(p)
$$

We have $\xi(t)=0$, and using the characteristic system one obtains

$$
\dot{\xi}(s)=D v(P(s)) \partial_{p} P(s)-D v(p) .
$$

So $\dot{\xi}(t)=0$. Differentiating once more, we get

$$
\ddot{\xi}(s)=D^{2} v(P(s)) \dot{P}(s) \partial_{p} P(s)+D v(P(s)) \partial_{p} \dot{P}(s) .
$$

As is easily checked, $D v(p)$ and $D^{2} v(p)$ are bounded independently of $p$, so that

$$
|\ddot{\xi}(s)| \leq C\left(|\dot{P}(s)|\left|\partial_{p} P(s)\right|+\left|\partial_{p} \dot{P}(s)\right|\right),
$$

and therefore by the characteristic system

$$
|\ddot{\xi}(s)| \leq C\left(\left|\partial_{p} P(s)\right|\left(\|E(s)\|_{\infty}+\|B(s)\|_{\infty}\right)+\left(\|\nabla E(s)\|_{\infty}+\|\nabla B(s)\|_{\infty}\right)\left|\partial_{p} X(s)\right|\right) .
$$


Resubstituting, we have

$$
\left|\partial_{p} X(s)\right| \leq|\xi(s)+(s-t) D v(p)|
$$

and

$$
\partial_{p} P(s)=(D v(P(s)))^{-1}\left(\dot{\xi}(s)+D_{p} v\right) .
$$

Assuming $|p| \leq P_{0}+1$, we can estimate

$$
\left|\partial_{p} P(s)\right| \leq C\left(P_{0}\right)(|\dot{\xi}(s)|+1) .
$$

Using the free streaming condition, we finally obtain the following second order differential inequality for $\xi$ :

$$
|\ddot{\xi}(s)| \leq C\left(P_{0}\right) \alpha\left\{(1+s)^{-3 / 2}+(t-s)(1+s)^{-5 / 2}+(1+s)^{-3 / 2}|\dot{\xi}(s)|+(1+s)^{-5 / 2}|\xi(s)|\right\}
$$

By Lemma 3.1,

$$
|\xi(s)| \leq C\left(P_{0}\right) \alpha(t-s) e^{C\left(P_{0}\right) \alpha},
$$

where we possibly have to adjust the constant $C\left(P_{0}\right)$. In terms of the characteristic variables this means for $\alpha$ chosen sufficiently small that

$$
\left|\partial_{p} X(0, t, x, p)+t D v(p)\right| \leq \epsilon t,
$$

where $\epsilon>0$ is prescribed such that

$$
\frac{|p \otimes p|}{1+|p|^{2}}+\epsilon \sqrt{1+|p|^{2}} \leq \beta<1 \text { for }|p| \leq P_{0}+1 .
$$

Here $p \otimes p$ denotes the matrix whose $(i, j)$-entry is $p_{i} p_{j}$. This implies

$$
\left|\partial_{p} X(0, t, x, p)+\frac{t}{\sqrt{1+|p|^{2}}} I\right| \leq \epsilon t+\left|\frac{t}{\sqrt{1+|p|^{2}}} I-t D v(p)\right| .
$$

By direct computation

$$
D v(p)=\frac{1}{\sqrt{1+|p|^{2}}}\left(I-\frac{p \otimes p}{1+|p|^{2}}\right)
$$

hence

$$
\left|\partial_{p} X(0, t, x, p)+\frac{t}{\sqrt{1+|p|^{2}}} I\right| \leq \epsilon t+\frac{t}{\sqrt{1+|p|^{2}}} \frac{|p \otimes p|}{1+|p|^{2}}<\frac{t}{\sqrt{1+|p|^{2}}} \beta .
$$

So the linear map $\partial_{p} X(0, t, x, p)$ is invertible, and in conclusion the transformation $\Psi: B_{P_{0}+1} \rightarrow \mathbb{R}^{3}, p \mapsto X(0, t, x, p)$ is a local diffeomorphism. It is even a diffeomorphism onto its image, since it is one-to-one as well. Let $p, \bar{p} \in B_{P_{0}+1}$ be given and $p_{\tau}:=$ $\tau p+(1-\tau) \bar{p}$. Then

$$
\begin{aligned}
|\Psi(p)-\Psi(\bar{p})| & =\left|\int_{0}^{1} \partial_{p} X\left(0, t, x, p_{\tau}\right)(p-\bar{p}) d \tau\right| \\
& =\left|\int_{0}^{1}\left[\partial_{p} X\left(0, t, x, p_{\tau}\right)+\frac{t}{\sqrt{1+\left|p_{\tau}\right|^{2}}} I\right](p-\bar{p}) d \tau-\int_{0}^{1} \frac{t}{\sqrt{1+\left|p_{\tau}\right|^{2}}}(p-\bar{p}) d \tau\right| \\
& \geq t|p-\bar{p}| \int_{0}^{1} \frac{1}{\sqrt{1+\left|p_{\tau}\right|^{2}}} d \tau-\beta t|p-\bar{p}| \int_{0}^{1} \frac{1}{\sqrt{1+\left|p_{\tau}\right|^{2}}} d \tau \\
& \geq C(1-\beta) t|p-\bar{p}|,
\end{aligned}
$$


where $C$ depends only on $P_{0}$.

Denote the open range of $\Psi$ by $U$ and let $\Phi: U \rightarrow B_{P_{0}+1}$ be its inverse. Calculation gives

$$
\begin{aligned}
\rho(t, x) & =\int_{B_{P_{0}+1}} f(t, x, p) d p \\
& =\int_{\Phi\left(\Psi\left(B_{P_{0}+1}\right)\right)} f^{\circ}(\Psi(p), P(0, t, x, p)) d p \\
& =\int_{\Psi\left(B_{P_{0}+1}\right)} f^{\circ}(w, P(0, t, x, \Phi(w)))|\operatorname{det} D \Phi(w)| d w .
\end{aligned}
$$

For the functional determinant showing up here we have by our previous calculations

$$
|\operatorname{det} D \Phi(w)|=\left|\operatorname{det}[D \Psi(\Phi(w))]^{-1}\right|=\frac{1}{|\operatorname{det} D \Psi(\Phi(w))|} .
$$

Note that

$$
\begin{aligned}
\operatorname{det} D \Psi(p) & =\operatorname{det}\left(\partial_{p} X(0, t, x, p)+\frac{t}{\sqrt{1+|p|^{2}}} I-\frac{t}{\sqrt{1+|p|^{2}}} I\right) \\
& =\frac{t^{3}}{\left(1+|p|^{2}\right)^{3 / 2}} \operatorname{det}\left(\sqrt{1+|p|^{2}} \frac{\partial_{p} X(0, t, x, p)+\frac{t}{\sqrt{1+|p|^{2}}} I}{t}-I\right) .
\end{aligned}
$$

But for the first matrix in the argument of the determinant we have

$$
\left|\sqrt{1+|p|^{2}} \frac{\partial_{p} X(0, t, x, p)+\frac{t}{\sqrt{1+|p|^{2}}} I}{t}\right| \leq \beta<1,
$$

so the absolute value of the determinant is bounded from below by a positive constant $C_{\beta}$. Returning to $(3.1)$, it is seen that

$$
|\operatorname{det} D \Phi(w)| \leq \frac{C_{\beta, P_{0}}}{t^{3}}
$$

resulting in

$$
\rho(t, x) \leq \frac{C R_{0}^{3}\left\|f^{\circ}\right\|_{\infty}}{t^{3}}
$$

In addition,

$$
|j(t, x)| \leq \rho(t, x) \leq \frac{C^{*}}{t^{3}} .
$$

For the bounds to be obtained for $\partial_{x} \rho$ and $\partial_{x} j$, note that

$$
\begin{aligned}
\left|\partial_{x} \rho(t, x)\right| & \leq C\left(P_{0}+1\right)^{3}\left\|\partial_{x} f(t)\right\|_{\infty}, \\
\left|\partial_{x} f(t, x, p)\right| & \leq C\left(\left|\partial_{x} X(0, t, x, p)\right|+\left|\partial_{x} P(0, t, x, p)\right|\right) .
\end{aligned}
$$

Next let $\xi(s):=\partial_{x} X(s, t, x, v)-I$ such that $\dot{\xi}(s)=D v(P(s)) \partial_{x} P(s)$. Obviously $\xi(t)=$ $\dot{\xi}(t)=0$. Differentiating further one has

$$
\begin{aligned}
|\ddot{\xi}(s)| & =\left|D^{2} v(P(s)) \dot{P}(s, t, x, p) \partial_{x} P(s, t, x, p)+D v(P(s)) \partial_{x} \dot{P}(s, t, x, p)\right| \\
& \leq \alpha C\left((1+s)^{-3 / 2}\left|\partial_{x} P(s, t, x, p)\right|+(1+s)^{-5 / 2}\left|\partial_{x} X(s, t, x, p)\right|\right),
\end{aligned}
$$


where again the decay of the fields due to (almost) free streaming was employed. By definition

$$
\left|\partial_{x} X(s) \leq\right| \xi(s) \mid+1 \quad \text { and } \quad\left|\partial_{x} P(s)\right| \leq C|\dot{\xi}(s)|
$$

and we may assume $|p| \leq P_{0}+1$ to discover the relation

$$
|\ddot{\xi}(s)| \leq C \alpha\left\{(1+s)^{-5 / 2}|\xi(s)|+(1+s)^{-3 / 2}|\dot{\xi}(s)|+\alpha(1+s)^{-5 / 2}\right\},
$$

which by Lemma 3.1 implies

$$
|\xi(s)| \leq C \alpha \int_{s}^{t}(1+\sigma)^{-3 / 2} d \sigma e^{C \alpha \int_{s}^{t}(1+\sigma)^{-3 / 2}} d \sigma \leq 2 C \alpha e^{2 C \alpha} .
$$

An easy application of Gronwall's Lemma shows that $|\dot{\xi}(s)|$ is bounded too, which means

$$
\left(\left|\partial_{x} X(0, t, x, p)\right|+\left|\partial_{x} P(0, t, x, p)\right|\right) \leq C,
$$

and all claims are proved.

\section{Decay of the fields}

Proof. [Proof of Proposition 2.4.] First let the constants $\alpha$ and $C_{1}$ be as given in Proposition 2.3. We want to get sufficiently good decay rate estimates for the fields from the field equations, the decay of the source terms, and the free streaming condition. The field $E_{T}$ is treated first. We have

$$
\Delta E_{T}=-\partial_{t}(\Delta A)=\partial_{t}(\mathbb{P} j)=\mathbb{P}\left(\partial_{t} j\right)
$$

where the last equation, i.e., the commutativity of $\mathbb{P}$ and $\partial_{t}$, is read off directly from the Fourier representation of the projection operator (compare with (4.1) or the Appendix.)

The Vlasov Equation, equation (1.1), then implies

$$
\partial_{t} j(t, x)=\int \partial_{t} f(t, x, p) v(p) d p=-\int\left\langle v(p), \nabla_{x} f\right\rangle v(p) d p-\int v(p) \otimes K(t, x, p) \nabla_{p} f d p
$$

Here $K(t, x, p)=E(t, x)+v(p) \times B(t, x)$. Integration by parts in the last term finally leads to

$$
\begin{aligned}
\partial_{t} j(t, x) & =-\int \operatorname{div}_{x}[f(t, x, p) v(p) \otimes v(p)] d p+\int \frac{I-v \otimes v}{\sqrt{1+p^{2}}} f(t, x, p) K(t, x, p) d p \\
& =: G_{1}(t, x)+G_{2}(t, x),
\end{aligned}
$$

where the divergence appearing is to be understood row-wise.

Writing $E_{T}=E_{T}^{1}+E_{T}^{2}$, where the components of the right hand side are solutions of $\Delta E_{T}^{1}=\mathbb{P}\left(G_{1}\right)$ and $\Delta E_{T}^{2}=\mathbb{P}\left(G_{2}\right)$ respectively, we treat each of them separately. Recall the Fourier representation of the projection operator $\mathbb{P}$ :

$$
\begin{gathered}
\mathbb{P} F(x)=\int e^{i k x} \frac{|k|^{2} I-k \otimes k}{|k|^{2}} \hat{F}(k) d k, \\
\hat{F}(k)=(2 \pi)^{-3} \int e^{-i k x} F(x) d x
\end{gathered}
$$


(see the Appendix). The solution of the Poisson equation may then be expressed as

$$
E_{T}^{l}(t, x)=-\int e^{i k x} \frac{|k|^{2} I-k \otimes k}{|k|^{4}} \hat{G}_{l}(k) d k, \quad l=1,2 .
$$

Introducing $M=\left(M_{1}, M_{2}, M_{3}\right)=\int f(t, x, p) v(p) \otimes v(p) d p$, it follows that

$$
\begin{aligned}
E_{T}^{1}(t, x) & =\int e^{i k x} \frac{\left|k^{2}\right| I-k \otimes k}{|k|^{4}} \hat{G}_{1}(t, k) d k \\
& =\sum_{j} \int e^{i k x} \frac{\left|k^{2}\right| I-k \otimes k}{|k|^{4}} i k_{j} \hat{M}_{j}(t, k) d k \\
& =i \sum_{j} \int e^{i k x} m_{j}(k) \hat{M}_{j}(t, k) d k,
\end{aligned}
$$

where $m_{j}(k)=\frac{\left|k^{2}\right| I-k \otimes k}{|k|^{3}} \frac{k_{j}}{|k|}$ is a function homogeneous of degree -1 . The theory of pseudodifferential operators (see [8, Lem. 2.4]) permits us to make the estimate:

$$
\left\|E_{T}^{1}(t)\right\|_{\infty} \leq C\left(\|M\|_{\infty}+\|M\|_{p}\right), \quad 1 \leq p<3 .
$$

Note that we also obtain a similar expression for $\nabla_{x} E_{T}^{1}(t)$, where the symbol of the the operator is now homogeneous of degree 0. Applying the last estimate given in the proof of Lemma 2.4 in [8] we obtain

$$
\left\|\nabla_{x} E_{T}^{1}(t)\right\|_{\infty} \leq C\left(\gamma^{3 / p^{\prime}-2}\|\nabla M\|_{p}+\log \left(\gamma^{-1}\right)\|M\|_{\infty}+\|M\|_{q}\right),
$$

$3<p<\infty, 1<q<\infty$. Here the parameter $\gamma$ is restricted to the interval $] 0,1]$. Since $\|M(t)\|_{\infty} \leq\|\rho(t)\|_{\infty} \leq C t^{-3}$, equation (4.3) implies that with $p=5 / 2$,

$$
\left\|E_{T}^{1}(t)\right\|_{\infty} \leq C t^{-9 / 5} .
$$

Using estimates from the proof of Proposition 2.3 and $\|\nabla M(t)\|_{\infty} \leq\|\nabla \rho(t)\|_{\infty} \leq C$ it follows that

$$
\|\nabla M(t)\|_{p} \leq\|\nabla M\|_{\infty}^{\frac{p-1}{p}}\|\nabla M\|_{1}^{1 / p} \leq C\|\nabla M\|_{1}^{1 / p} \leq C t^{3 / p},
$$

so that setting $\gamma=t^{-3}$ in (4.4) we get for $t \geq 1$

$$
\left\|\nabla E_{T}^{1}(t)\right\|_{\infty} \leq C\left(t^{-3(1-3 / p)+3 / p}+t^{-3} \log t+t^{-3(q-1)}\right) \leq C t^{-8 / 3},
$$

where the choice $p=36, q=17 / 9$ was made.

Now consider $\Delta E_{T}^{2}=\mathbb{P}\left(G_{2}\right)$. We have

$$
\begin{array}{r}
E_{T}^{2}(t, x)=\int e^{i k x} \frac{|k|^{2} I-k \otimes k}{|k|^{4}} \hat{G}_{2}(t, k) d k=\int e^{i k x} m_{-2} \hat{G}_{2}(t, k) d k, \\
\partial_{j} E_{T}^{2}(t, x)=i \int e^{i k x} \frac{|k|^{2} I-k \otimes k}{|k|^{3}} \frac{k_{j}}{|k|} \hat{G}_{2}(t, k) d k=\int e^{i k x} m_{-1} \hat{G}_{2}(t, k) d k .
\end{array}
$$

The symbols $m_{\alpha}$ showing up here are homogeneous of degree $\alpha$. A simple adaptation of the proof of (45) in [8] shows that

$$
\left\|E_{T}^{2}(t)\right\|_{\infty} \leq C\left(\left\|G_{2}(t)\right\|_{\infty}+\left\|G_{2}(t)\right\|_{p}\right), \quad 1 \leq p<3 / 2,
$$


and as before

$$
\left\|\partial_{x} E_{T}^{2}(t)\right\|_{\infty} \leq C\left(\left\|G_{2}(t)\right\|_{\infty}+\left\|G_{2}(t)\right\|_{p}\right), \quad 1 \leq p<3 .
$$

Since $\left|G_{2}(t, x)\right| \leq\|K(t)\|_{\infty} \rho(t, x)$, we have

$$
\left\|G_{2}(t)\right\|_{5 / 4} \leq C(1+t)^{-3 / 2}\|\rho(t)\|_{\infty}^{1 / 5}\|\rho(t)\|_{1}^{4 / 5} \leq t^{-21 / 10}
$$

and

$$
\left\|G_{2}(t)\right\|_{5 / 2} \leq C(1+t)^{-3 / 2}\|\rho(t)\|_{\infty}^{3 / 5}\|\rho(t)\|_{1}^{2 / 5} \leq t^{-33 / 10} .
$$

Altogether this implies

$$
\begin{aligned}
\left\|E_{T}(t)\right\|_{\infty} & \leq C t^{-9 / 5}, \\
\left\|\nabla_{x} E_{T}(t)\right\|_{\infty} & \leq C t^{-8 / 3}, \quad t \geq 1 .
\end{aligned}
$$

Now we come to the other fields. The longitudinal part $E_{L}$ of the electric field is treated exactly as in the case of the Vlasov-Poisson system (cf. [10]):

$$
\begin{aligned}
\left\|E_{L}(t)\right\|_{\infty} & \leq C t^{-2} \\
\left\|\partial_{x} E_{L}(t)\right\|_{\infty} & \leq C t^{-3} \log t .
\end{aligned}
$$

The bounds for the magnetic field $B=\nabla \times A$ field are obtained in a way analogous to the procedure used so far. First we have a representation

$$
\nabla A(t, x)=\int e^{i k x} m_{-1}(k) \hat{j}(t, k) d k,
$$

with $m_{-1}$ homogeneous of degree -1 . Therefore

$$
\|B(t)\|_{\infty} \leq\|j(t)\|_{\infty}+\|j(t)\|_{5 / 2} \leq C t^{-9 / 5} .
$$

In analogy to our treatment of $E_{T}$ we find

$$
\left\|\nabla_{x} B(t)\right\|_{\infty} \leq C\left(\gamma^{3 / p^{\prime}-2}\|\nabla j\|_{p}+\log \left(\gamma^{-1}\right)\|j\|_{\infty}+\|j\|_{q}\right),
$$

and the proof may be completed as shown before.

\section{Continuous dependence}

In this section we denote by $C$ a constant depending only on $R_{0}, P_{0}$, which may change from line to line. For the proof we first collect some facts.

Let $\left(f, E_{L}, E_{T}, B\right)$ be a solution of the VD system on some time interval $[0, T[$ with $f_{0}=f(0) \in \mathcal{D}$. Define

$$
Q(t):=\sup \{|p|: \exists x, 0 \leq s \leq t: f(t, x, p) \neq 0\} .
$$

Then we have the following

Lemma 5.1. Let $f$ be a solution with $f(0) \in \mathcal{D}$. Then there holds

$$
\begin{aligned}
\|\rho(t)\|_{4 / 3}+\|j(t)\|_{4 / 3} & \leq C\left\|f_{0}\right\|_{\infty} \\
\|A(t)\|_{\infty} & \leq C\left\|f_{0}\right\| Q(t)^{1 / 3} \\
\|\nabla A(t)\|_{\infty}+\|\nabla \Phi(t)\|_{\infty} & \leq C\left\|f_{0}\right\|_{\infty} Q(t)^{5 / 3}
\end{aligned}
$$


To estimate the field $E_{T}$ only a local result is available.

Lemma 5.2. Let $f:[0, T] \times \mathbb{R}^{6} \rightarrow \mathbb{R}$ be a solution with $f(0) \in \mathcal{D}$. Then

$$
\left\|E_{T}(t)\right\|_{\infty, B_{R_{0}+T}} \leq C_{R_{0}+T}\left(1+\|\rho(t)\|_{3}\right)\left(\|F(t)\|_{6 / 5}+\|F(t)\|_{2}\right),
$$

where $F=F_{1}+F_{2}$ with

$$
\begin{aligned}
& F_{1}(t, x)=\int \operatorname{div}_{(x)}(f(t, x, p) v(p) \otimes v(p)) d p \\
& F_{2}(t, x)=\int \frac{I-v(p) \otimes v(p)}{\sqrt{1+|p|^{2}}} f(t, x, p)\left(E_{L}(t, x)+v(p) \times B(t, x)\right) d p .
\end{aligned}
$$

Detailed proofs of these lemmas are given in Pallard's paper [9], where the following theorem is also proved.

THEOREM 5.3. Let $f_{0} \in C^{2}\left(\mathbb{R}^{6}\right)$. Then there exists $T^{*}>0$ and a unique solution $\left(f, E_{L}, E_{T}, B\right)$ to the Vlasov-Darwin system with $f(0)=f_{0}$ satisfying

$$
\begin{aligned}
f & \in C^{1}\left(\left[0, T^{*}\left[\times \mathbb{R}^{3} \times \mathbb{R}^{3}\right),\right.\right. \\
E_{L}, B & \in C^{1}\left(\left[0, T^{*}\left[\times \mathbb{R}^{3}\right),\right.\right. \\
E_{T}, \nabla_{x} E_{T} & \in C\left(\left[0, T^{*}\left[\times \mathbb{R}^{3}\right),\right.\right.
\end{aligned}
$$

and such that for any $t \in\left[0, T^{*}[\right.$ the distribution function $f(t, \cdot)$ is compactly supported.

By inspection of the constants in the proof one finds that a strict lower bound for $T^{*}$ is given by $T^{\prime}:=\left(C\left\|f_{0}\right\|_{\infty}^{2}\right)^{-1}$, with a constant $C$ independent of $f_{0}$, i.e., $T^{*}>T^{\prime}$. In addition one has

$$
Q(t) \leq C\left(R_{0}, P_{0}\right) \quad\left(0 \leq t \leq T^{\prime}\right)
$$

One last ingredient for the proof of Proposition 2.5 is contained in the following.

LEMMA 5.4. Let $f$ be a solution and let $T^{\prime}$ be defined as above. Then

$$
\left\|\nabla_{x, p} f(t)\right\|_{\infty} \leq C\left(R_{0}, P_{0}\right) \quad\left(0 \leq t \leq T^{\prime}\right)
$$

The proof can again be found in [9]. From the Lemma we immediately deduce the bounds

$$
\|\nabla \rho(t)\|_{\infty}+\|\nabla j(t)\|_{\infty} \leq C, \quad 0 \leq t \leq T^{\prime}
$$

With these tools at hand we are now ready for the

Proof. [Proof of Proposition 2.5.] Let $\epsilon, T>0$ be given. From the above facts one immediately finds that the solution interval can be made as long as we wish and that $\left\|E_{L}(t)\right\|_{\infty}$ and $\|B(t)\|_{\infty}$ can be made as small as necessary by choosing $\delta$ sufficiently small.

It is standard to obtain a bound for $\left\|\nabla_{x} E_{L}(t)\right\|_{\infty}$ (see [10]), and for $\|\nabla B(t)\|_{\infty}$ we can use equation (4.7) from section 4. By finite propagation speed and since $\|\nabla j(t)\|_{\infty}$ 
remains bounded for $t \in\left[0, T^{\prime}\right]$, we can choose the parameter $\gamma$ on the right-hand side of the inequality properly to get the result.

We still have to get control over $\left\|E_{T}\right\|_{\infty}$ and $\left\|\nabla E_{T}\right\|_{\infty}$. From (4.3) and (4.5) we have the estimate

$$
\left\|E_{T}(t)\right\|_{\infty} \leq C\left(\|\rho(t)\|_{\infty}+\|\rho(t)\|_{2}+\left\|G_{2}(t)\right\|_{\infty}+\left\|G_{2}(t)\right\|_{5 / 4}\right)
$$

where the notation introduced in section 4 is used again. Now

$$
\begin{aligned}
\left|G_{2}(t, x)\right| & \leq\left|\int \frac{I-v(p) \otimes v(p)}{\sqrt{1+|p|^{2}}} f(t, x, p) K(t, x, p) d p\right| \\
& \leq C \int_{|p| \leq Q(t)} f(t, x, p)|K(t, x, p)| d p \\
& \leq C Q(t)^{3}\left\|f_{0}\right\|_{\infty}\|K(t)\|_{\infty, B_{R_{0}+T}} \chi_{B_{R_{0}+T}}(x),
\end{aligned}
$$

and since we have bounds for $\|K(t)\|_{\infty, B_{R_{0}+T}}$ by Lemma 5.2 , we get $\left\|E_{T}(t)\right\|_{\infty} \leq \epsilon$ for $\left\|f_{0}\right\|_{\infty}$ chosen sufficiently small in view of (5.1).

To estimate $\left\|\nabla E_{T}(t)\right\|$ we note as in section 4 :

$$
\left\|\nabla_{x} E_{T}(t)\right\|_{\infty} \leq C\left(\gamma^{3 / p^{\prime}-2}\|\nabla M\|_{p}+\log \left(\gamma^{-1}\right)\|M\|_{\infty}+\|M\|_{2}+\left\|G_{2}(t)\right\|_{\infty}+\left\|G_{2}(t)\right\|_{2}\right),
$$

with $0<\gamma \leq 1,3<p<\infty$. So again each term can be made as small as we wish, and the proof is complete.

\section{Proof of the theorem}

Proof. We start by choosing a constant $T_{0}>0$ such that for $t \geq T_{0}$ the following holds:

$$
C_{2} t^{-9 / 5}<\alpha(1+t)^{-3 / 2} \quad \text { and } \quad C_{2} t^{-8 / 3}<\alpha(1+t)^{-5 / 2},
$$

where $\alpha$ and $C_{2}$ are the constants given by Proposition 2.4. Proposition 2.5 says that there exists $\delta>0$ such that a solution of the Vlasov-Darwin system with initial $f_{0} \in \mathcal{D}$ and $\left\|f_{0}\right\|_{\infty}<\delta$ satisfies

$$
\begin{gathered}
\left\|E_{L}(t)\right\|_{\infty}+\left\|E_{T}(t)\right\|_{\infty}+\|B(t)\|_{\infty}+\left\|\nabla E_{L}(t)\right\|_{\infty}+\left\|\nabla E_{T}(t)\right\|_{\infty}+\|\nabla B(t)\|_{\infty} \\
<\alpha\left(1+T_{0}\right)^{-5 / 2}
\end{gathered}
$$

for $t$ belonging to $\left[0, T_{0}+1\right]$. Moreover, it may be assumed that the maximal interval of existence $I=\left[0, T_{\max }\left[\right.\right.$ is strictly larger than $\left[0, T_{0}+1\right]$, i.e. $T_{0}+1<T_{\max }$.

If $f$ is a solution as above, then by continuity $f$ satisfies a free streaming condition with parameter $\alpha$ on an interval $\left[0, T^{*}\right]$, with $T_{0}<T^{*} \leq T_{\max }$, and $T^{*}$ may be chosen maximal with these properties. Because of equation (6.1) we may now conclude with Proposition 2.4 that

$$
\begin{array}{r}
\left\|E_{L}(t)\right\|_{\infty}+\left\|E_{T}(t)\right\|_{\infty}+\|B(t)\|_{\infty} \leq \alpha(1+t)^{-3 / 2}, \\
\left\|\nabla E_{L}(t)\right\|_{\infty}+\left\|\nabla E_{T}(t)\right\|_{\infty}+\|\nabla B(t)\|_{\infty} \leq \alpha(1+t)^{-5 / 2},
\end{array}
$$

for all $t \in I$. But this implies that $T_{\max }=\infty$ and the solution is global. 


\section{Spherically symmetric initial data}

In the case where the initial datum $f^{\circ}$ is spherically symmetric, which in the present situation by definition means

$$
f^{\circ}(Q x, Q p)=f^{\circ}(x, p) \quad \forall x, p \in \mathbb{R}^{3}, Q \in O(3),
$$

the Vlasov-Darwin system reduces to the relativistic Vlasov-Poisson system, as is shown in the following. First we show that spherical symmetry is preserved.

Lemma 7.1. Let $f:\left[0, T\left[\times \mathbb{R}^{6} \rightarrow \mathbb{R}\right.\right.$ be a classical solution of the Vlasov-Darwin system and let $f(0)$ be spherically symmetric. Then $f(t)$ is spherically symmetric for all $0 \leq t<T$.

Actually we will see that any invariance of the initial datum with respect to an orthogonal transformation is preserved for all times, which implies at the same time that e.g. cylindrical symmetry or reflectional symmetries are preserved as well.

Proof. Let $Q \in O(3)$ be given and set $\tilde{f}(t, x, p):=f(t, Q x, Q p)$. It suffices to show that $\tilde{f}$ solves the Vlasov-Darwin system. One finds

$$
\begin{aligned}
\tilde{\rho}(t, x) & :=\int \tilde{f}(t, x, p) d p=\rho(t, Q x), \\
\tilde{j}(t, x) & :=\int \tilde{f}(t, x, p) v(p) d p=Q^{-1} j(t, Q x) .
\end{aligned}
$$

For the potentials $\tilde{\Phi}$ and $\tilde{A}$ one therefore has

$$
\begin{aligned}
& \tilde{\Phi}(t, x)=\Phi(t, Q x), \\
& \tilde{A}(t, x)=Q^{-1} A(t, Q x),
\end{aligned}
$$

as can be seen easily from the Fourier representation of the projection operator $\mathbb{P}$, see (4.1). This implies that

$$
\begin{aligned}
& \tilde{E}_{L}(t, x):=\nabla \tilde{\Phi}(t, x)=Q^{-1} E_{L}(t, Q x), \\
& \tilde{E}_{T}(t, x):=-\partial_{t} \tilde{A}(t, x)=Q^{-1} E_{T}(t, Q x) .
\end{aligned}
$$

We set $\tilde{B}:=\nabla \times \tilde{A}$. Then by Lemma 2.3 in [9] and since

$$
\partial_{t} \tilde{\rho}(t, x)+\nabla \cdot \tilde{j}(t, x)=\partial_{t} \rho(t, Q x)+\nabla \cdot j(t, Q x)=0
$$

the quantities $\left(E_{L}, E_{T}, B\right)$ solve the field equation part of the Vlasov-Darwin system.

We have to show that the transport equation (1.1) holds. Consider the term $p \times \tilde{B}(t, x)=p \times(\nabla \times \tilde{A}(t, x))$. By well known vector identities we can write

$$
\begin{aligned}
p \times \tilde{B}(t, x) & =\nabla(\tilde{A}(t, x) p)-(p \cdot \nabla) \tilde{A}(t, x)) \\
& =\left[(D \tilde{A}(t, x))^{t}-D \tilde{A}(t, x)\right] p .
\end{aligned}
$$

Here $D$ denotes the total derivative with respect to $x$. Now $D \tilde{A}(t, x)=$ $Q^{-1} D A(t, Q x) Q$ and therefore

$$
\begin{aligned}
Q(p \times \tilde{B}(t, x)) & =\left[(D A(t, Q x))^{t}-D A(t, Q x)\right] Q p \\
& =Q p \times B(t, Q x) .
\end{aligned}
$$


The last equality holds because the forgoing applies equally well to $A$ as to $\tilde{A}$. This finally leads to (1.1).

We have seen that spherical symmetry is preserved for all times. This includes that for $t \in[0, T[, Q \in O(3)$ the following identities hold

$$
\begin{aligned}
\rho(t, Q x) & =\rho(t, x), \\
\Phi(t, Q x) & =\Phi(t, x), \\
j(t, Q x) & =Q j(t, x), \\
A(t, Q x) & =Q A(t, x .)
\end{aligned}
$$

LEMMA 7.2. The vector field $j$ is radial.

Proof. In the following the dependence of $j$ on $t$ is suppressed. Let $x \in \mathbb{R}^{3} \backslash\{0\}$ be given and choose a positive orthonormal basis $\left(b_{1}, b_{2}, b_{3}\right)$ with $b_{1}=\frac{x}{|x|}$. Let $Q_{1}, Q_{2}$ be orthogonal transformations with matrices

$$
M_{1}=\left(\begin{array}{ccc}
-1 & 0 & 0 \\
0 & -1 & 0 \\
0 & 0 & 1
\end{array}\right), \quad M_{2}=\left(\begin{array}{ccc}
-1 & 0 & 0 \\
0 & 1 & 0 \\
0 & 0 & -1
\end{array}\right),
$$

with respect to the basis chosen. Let $j(x)=\sum_{j} \alpha_{j} b_{j}$. Then

$$
Q_{1} j(x)=-\alpha_{1} b_{1}-\alpha_{2} b_{2}+\alpha_{3} b_{3}, \quad Q_{2} j(x)=-\alpha_{1} b_{1}+\alpha_{2} b_{2}-\alpha_{3} b_{3} .
$$

But since $Q_{1} j(x)=j\left(Q_{1} x\right)=j(-x)=j\left(Q_{2} x\right)=Q_{2} j(x)$ it follows that $\alpha_{2}=\alpha_{3}=0$.

Lemma 7.3. There holds $\mathbb{P} j \equiv 0$.

Proof. First note, that $\nabla \cdot j(Q x)=\nabla \cdot j(x)$ for all $Q \in O(3)$. Recall the definition of $\mathbb{P}$ :

$$
\mathbb{P} j(x)=j(x)+\nabla \Psi(x)
$$

where

$$
\Psi(x)=\frac{1}{4 \pi} \int \frac{\nabla \cdot j(y)}{|x-y|} d y .
$$

But since the source term $\nabla \cdot j$ has rotational symmetry, the foregoing simplifies to

$$
\nabla \Psi(x)=-\int_{0}^{r} s^{2}(\nabla \cdot j)(s) d s \frac{x}{r^{3}}, \quad r=|x| .
$$

The integral in the last expression can be transformed to

$$
\begin{aligned}
\int_{0}^{r} s^{2}(\nabla \cdot j)(s) d s & =\frac{1}{4 \pi} \int_{B_{r}} \nabla \cdot j d V \\
& =\frac{1}{4 \pi} \int_{\partial B_{r}} j n d S \\
& =j(x) n r^{2}
\end{aligned}
$$

So

$$
\mathbb{P} j(x)=j(x)+\nabla \Psi(x)=j(x)-j(x) n \frac{x}{r}=0 .
$$


Lemma 7.3 implies that also $A(t)=0$ for $t \in[0, T[$. This immediately leads to $E_{T}=B=0$, so that the following proposition is proved.

Proposition 7.4. In the case of a spherically symmetric initial datum $f^{\circ}$, the VlasovDarwin system reduces to the (spherically symmetric) relativistic Vlasov-Poisson system (with repelling forces). Hence in this case the solution is global.

The proof of the last statement is given in [6].

Appendix. We start with some remarks about the projection operator $\mathbb{P}: L^{2}\left(\mathbb{R}^{3}\right) \rightarrow L^{2}\left(\mathbb{R}^{3}\right)$, which is defined as follows. For $F \in C_{c}^{1}\left(R^{3} ; R^{3}\right)$, one sets

$$
\begin{aligned}
(\mathbb{P} F)(x) & =F(x)+\nabla \Phi(x), \quad \text { where } \\
\Phi(x) & :=\frac{1}{4 \pi} \int \frac{(\nabla \cdot F)(y)}{|x-y|} d y .
\end{aligned}
$$

Since $-\Delta \Phi=\nabla \cdot F$ we clearly obtain $\nabla \cdot \mathbb{P} F=0$. Applying the Fourier transform to these relations it follows that

$$
\begin{aligned}
\hat{P} F(\xi) & =\hat{F}(\xi)+i \hat{\Phi}(\xi) \xi, \\
\hat{\Phi}(\xi) & =\frac{i}{|\xi|^{2}} \xi \cdot \hat{F}(\xi),
\end{aligned}
$$

hence

$$
\hat{\mathbb{P} F}(\xi)=\left(I-\frac{\xi \otimes \xi}{|\xi|^{2}}\right) \hat{F}(\xi) .
$$

Therefore $|\hat{P} F(\xi)| \leq C|\hat{F}(\xi)|$, so that by the Plancherel Theorem $\mathbb{P}$ extends to a continuous operator on $L^{2}\left(\mathbb{R}^{3}\right)$ characterized by (A.1).

We conclude with some remarks about the pseudodifferential operators used in section 4. Such an operator is of the form

$$
A u(x)=\frac{1}{(2 \pi)^{n}} \int A_{0}(\xi) \hat{u}(\xi) e^{i x \cdot \xi} d \xi,
$$

where $n$ is the dimension of the underlying space $\mathbb{R}^{n}$, the function $A_{0}$ is called the symbol of the operator and is chosen from a suitable set of functions, and $\hat{u}$ is the Fourier transform of $u$. We can restrict ourselves to the case that $u$ belongs to the Schwarz space $\mathcal{S}\left(\mathbb{R}^{n}\right)$ of rapidly decreasing functions. It is shown, e.g., in [5], that an operator of the form (A.2) with a symbol $A_{0}$ homogeneous of degree $\alpha>-n$, i.e. $A_{0}(t \xi)=t^{\alpha} A_{0}(\xi)$ for $t>0, \xi \in \mathbb{R}^{n}$, has a representation as an integral operator of the form

$$
A u(x)=\int a_{0}(x-y) u(y) d y,
$$

where $a_{0}$ is a function homogeneous of degree $-\alpha-n$. For such a (smooth) function one clearly has

$$
\left|a_{0}(y)\right| \leq C|y|^{-\alpha-n}
$$

and this is all one needs to know for the estimates presented here and in [8].

Acknowledgement. I would like to thank Prof. G. Rein for bringing me in contact with this interesting topic and for many valuable discussions. 


\section{REFERENCES}

[1] C. Bardos and P. Degond, Global existence for the Vlasov-Poisson system in 3 space variables with small initial data, Ann. Inst. H. Poincare Anal. Non Lineaire, 2, 101-118, 1985.

[2] C. Bardos, H.T. Ngoan and P. Degond, Existence globale des solutions des équations de VlasovPoisson relativistes en dimension 3, C.R. Acad. Sci. Paris Sr. I Math. 301 (1985), (6), 265-268, 1985.

[3] S. Benachour, F. Filbet, P. Laurençot and E. Sonnendrücker, Global existence for the VlasovDarwin system in $\mathbb{R}^{3}$ for small initial data, Math. Meth. Appl. Sci., 26, 297-319, 2003.

[4] N. Besse, N. Mauser and E. Sonnendrücker, Numerical approximation of self consistent Vlasov models for low-frequency electromagnetic phenomena, Int. J. Appl. Math. Comput. Sci., 17(3), 361-374, 2007.

[5] G.I. Eskin, Boundary Value Problems for Elliptic Pseudodifferential Equations, AMS, Providence, 1980.

[6] R.T. Glassey and W.A. Strauss, On symmetric solutions of the relativistic Vlasov-Poisson system, Comm. Math. Phys., 101, 459-473, 1985.

[7] R.T. Glassey and W.A. Strauss, Absence of shocks in an initially dilute collisionless plasma, Comm. Math. Phys., 113, 191-208, 1987.

[8] S. Klainerman and G. Staffilani, A new approach to study the Vlasov-Maxwell system, Commun. Pure Appl. Anal., 1(1), 103-125, 2002.

[9] C. Pallard, The initial value problem for the relativistic Vlasov-Darwin system, Int. Mat. Res. Not., 2006.

[10] G. Rein, Collisionless kinetic equations from astrophysics-the Vlasov-Poisson system, Handbook of Differential Equations, Evolutionary Equations, 3, 2007.

[11] G. Rein and A.D. Rendall, Global existence for solutions of the spherically symmetric VlasovEinstein system with small initial data, Comm. Math. Phys., 150, 561-583, 1992.

[12] H. Schmitz and R. Grauer, Darwin-Vlasov simulations of magnetized plasmas, arXiv:physics/0601220v1, 2006. 